\title{
Validation of Activation Recovery Interval in Structurally Normal Human Ventricles by Optical Mapping
}

\author{
P Langfield ${ }^{1}$ J Duchateau ${ }^{1,2}$, R Walton ${ }^{1}$, ME Martinez ${ }^{1}$, S Charron $^{1}$, J Magat ${ }^{1}, \mathrm{C} \mathrm{Cros}^{1}$, D Benoist ${ }^{1}$, \\ C Pascarel-Auclerc ${ }^{1}$, P Pasdois ${ }^{1}$, F Vaillant ${ }^{1}$, S Chaigne ${ }^{1}$, L Pourteau ${ }^{1}$, M Constantin $^{1}$, VN Dubes ${ }^{1}$, \\ P Diolez $^{1,3}$, B Stuyvers ${ }^{1,4}$, B Quesson ${ }^{1,3}$, F Sacher $^{1,2}$, L Labrousse $^{1,2}$, M Hocini $^{1,2}$, F Brette $^{1}$, \\ M Haissaguerre ${ }^{1,2}$, O Bernus ${ }^{1}$, E Vigmond $^{1}$ \\ ${ }^{1}$ IHU Liryc, Electrophysiology and Heart Modeling Institute, fondation Bordeaux Universit, F-33600 \\ Pessac- Bordeaux, France \\ ${ }^{2}$ Bordeaux University Hospital (CHU), Electrophysiology and Ablation Unit, F-33600 Pessac, \\ France \\ ${ }^{3}$ Univ Bordeaux, Centre de recherche Cardio-Thoracique de Bordeaux, U1045, F-33000, Bordeaux, \\ France \\ ${ }^{4}$ Memorial University of Newfoundland, Canada
}

\begin{abstract}
Background: A large Dispersion of Repolarization $(D o R)$ is associated with an increased arrhythmogenic risk. This can be measured clinically by calculating the Activation Recovery Interval (ARI) to estimate Action Potential Duration (APD). However, the ability of ARI to accurately predict $A P D$ dispersion in patients with repolarization abnormality has not been determined.

Objective: Compare ARI calculated from patients with optical mapping of human hearts to establish the validity of $A R I$ as a surrogate for $A P D$.

Methods: Optical mapping (OM) was performed on the left ventricles of 4 explanted human hearts. APD and repolarization times were measured endo- and epicardially on the anterior of the LV. Electroanatomic mapping was performed with CARTO over the entire endo- and epicardial surfaces of 3 patients. Activation and repolarization were calculated, dispersion of ARI was measured.

Results: APD and ARI were consistent between mapping methods over most of the sub-regions studied. Epicardium ARI dispersion was consistently higher than that of the endocardium in both OM and CARTO datasets.

Conclusion: APD distribution, and consequently DoR, agree between mapping methods. Measuring DoR by ARI accurately assesses the underlying repolarization abnormalities in patients.
\end{abstract}

\section{Introduction}

The morphology of unipolar electrograms provides a way of accessing the precise spatio-temporal detail of underlying electrical signals that coordinate the function of the heart, without the need for highly-invasive surgery.

These electrical signals are formed by action potentials in cardiac myocytes, whereby the potential of the cell quickly raises (depolarization), plateaus and then slowly falls (repolarization) and returns to a resting state. The cells are generally at a resting state, where a nearby stimulus can cause them to depolarize. The high potential provides a stimulus that triggers neighboring cells causing them to depolarize and undergo an action potential leading to a chain of subsequent action potentials one after another in the form of a wave. While the cell is repolarizing, it cannot depolarize, which allows the cell - and eventually the chain of cells, to return to the resting state. This also forms a wave block that prevents the wave of activity from traveling backwards.

The timing of repolarization across the cells is very important in the maintenance of correct conduction pathways. A large dispersion of repolarization has been linked with fibrillation and arrhythmia [1]. Regions of cells that repolarize too early can become vulnerable to early activation, for example via ectopic beats, which can initiate irregular signals that interfere with the regular function of the heart. Later repolarizing regions can form wave block that can redirect the pathway, and can also lead to early activation. Unfortunately, measuring repolarization times is difficult without highly invasive procedures. 
However, in the ventricles, the depolarization and repolarization times have been approximated via markers in unipolar electrogram signals (UE), such as in the case of calculating activation recovery intervals (ARI) [2]. As a marker for depolarization, the moment of steepest negative slope during the activation phase is widely accepted [2]. Repolarization is often identified using the Wyatt method [3], which identifies this moment as the steepest positive slope during the $\mathrm{T}$ wave, however this description is more disputed. The ARI is defined as the time between local depolarization time and repolarization time according to these markers.

The aim of this study is to validate this approach of computing repolarization times by comparing spatial heterogeneity of algorithmically calculated ARI from unipolar electrograms, with optically recorded APD in ventricles from explanted hearts. Additionally, we present our detailed methodology for comparing two very different datasets from different sources.

\section{Methods}

\subsection{CARTO ARI calculations}

The 3 human heart CARTO datasets comprise a ventricular mesh (with no septum), and a set of $2500 \mathrm{msec}$ unipolar recordings from electrode sites across the endo- and epicardium walls of the mesh. The signals are recorded in batches of roughly ten, and associated to each batch is the set of 12-lead ECG signals that were recording concurrently. Patient 1 has been diagnosed with Idiopathic VF and patients 2 and 3 with Brugada syndrome. The signals undergo a bandpass filter allowing frequencies above $0.5 \mathrm{~Hz}$ and below $50 \mathrm{~Hz}$ to remove baseline wander and power supply noise.

We identified the prominent peaks in each of the V5 leads, which correspond to the global depolarization phase across the heart. Around each peak we define an interval in which to identify the local depolarization time in the unipolar signal. We use the peak of the V5 lead since this is consistently a clear marker that is typically unambiguous when computed algorithmically. Any ambiguity in identifying the V5 peaks provides an effective criteria for filtering irregular signals. In addition, this marker is a global event that occurs in each local UE signal. Hence we can align the UE signals by this common event, which can be used to consider local times times of features of the UE signals on a global scale, for example, in order to study dispersion of depolarization or repolarization times.

The local depolarization time was chosen as the point with maximum negative derivative in an interval $[-25,60]$ msec about the V5 peak. The $2500 \mathrm{msec}$ unipolar recordings typically exhibit 2 to 3 beats, but the last beat is consistently the clearest and typically finishes before the ac- tivation of the next beat occurs. Hence, we define the interval for repolarization as $240 \mathrm{msec}$ after the last V5 peak to the end of the signal to incorporate the $\mathrm{T}$ wave. The moment of repolarization was chosen as the maximum positive slope in this interval according to the Wyatt method [3]. ARI is calculated as the time from the moment of activation to the moment of repolarization.

\subsection{Optical APD calculations}

We obtained APD measurements via optical recordings from four explanted human hearts, one diagnosed as hypertrophy, another as concentric hypertrophy, and two healthy. Each set is in the form of two $100 \times 100$-pixel videos showing action potentials in the endo- and epicardium of a section of left ventricle (LV) free wall that was coated in a voltage-dependent dye and stimulated. For each pixel in the video the moment of depolarization was computed as the maximal derivative of the upstroke of the action potential. The repolarization time was computed as the moment the action potential recovers by $80 \%$ of its amplitude. The APD is the difference between these two times.

\subsection{Comparing the datasets}

In order to compare the two datasets directly, the both datasets were mapped to the same comparison region. The slices of optically recorded LV correspond to a subset of the CARTO ventricular meshes, and so this comparison region was chosen to be the region of $\mathrm{LV}$ common to all four slices in the cases of the endo- and epicardium.

To compare the slices, we first assigned universal ventricular coordinates (UVCs) [4] to each point. However, as they are slices, assigning UVCs via the computation described in the article [4] that solves the Laplacian, cannot be applied here. Hence, the UVCs were assigned by eye. We then identified regions of the LV endo- and epicardium that were common to all four slices. The APD data for each slice was then interpolated (linearly) to this common region so that the four slices correspond to the same resolution.

We first mapped the CARTO ARIs to the entire mesh using inverse distance weighted interpolation with exponent $p=5$, where the distances between meshpoints were geodesic rather than the Euclidean, which were calculated across the mesh via solutions of the Eikonal problem. UVCs were then calculated across the mesh, which were then used to map the ARIs to the common region via linear interpolation.

To identify regions of interest for comparison, we assigned a segment number according to the AHA 17segment left ventricle plan [5] using the LV rotational coordinate and the axicobasal coordinate from the UVC system. The comparison region and the segments can be seen 


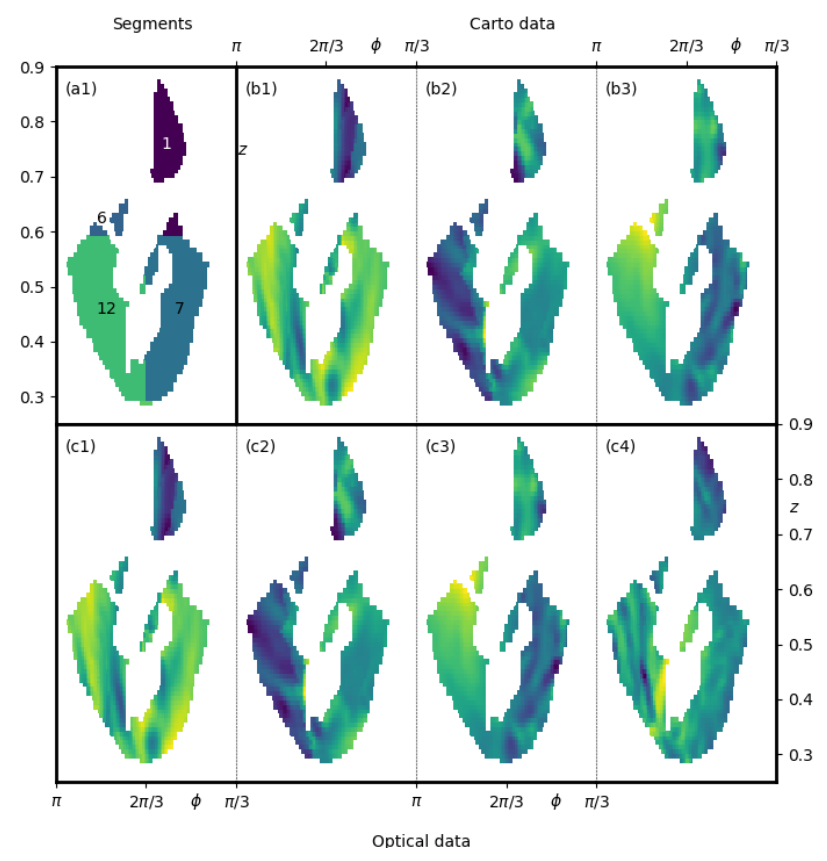

Figure 1. (a) Portion of left ventricle endocardium in UVC $(\phi, z)$-coordinates, with AHA 17-segment left ventricle plan segments labeled. APD (b) and ARI (c) data from optical and CARTO datasets, respectively, mapped to the portion of LV shown in (a).

in Figures 1 and 2 with the segments labeled; clearly only regions $1,6,7,12$ and 13 are of interest. Then the dispersion was measured over the endo- and epicardium for the ARI and APD data over the entire region and in the 5 different AHA segments shown.

\section{Results}

Table 1 shows the dispersion in ARI for each of the CARTO patients. This was calculated across the whole endo- and epicardium regions and across the individual segments. Table 2 shows the dispersion of APD for each of the slices of optically recorded heart. The mean for each region across each of the two walls separately and together is shown a the bottom. The ARI and APD data for the endo- and epicardium are shown in Figs 1 and 2 (a)

In general, the measures of dispersion across the epicardium are larger than those of the endocardium, which is consistent between the two datasets. However, the means associated with the CARTO dataset appear to be a factor of roughly $1.5 \times$ larger those of the optical dataset.

Generally, the dispersion is ordered according the the number of mesh points in each region. But some clear exceptions occur.

\begin{tabular}{|c|c|c|c|c|c|}
\hline : & $\frac{0}{8}$ & 1 & 6 & 7 & 12 \\
\hline $1_{\text {endo }}$ & 177.1 & 61.9 & 155.5 & 117.3 & 154.9 \\
\hline $1_{e p i}$ & 317.5 & 247.3 & 81.6 & 282.4 & 248.7 \\
\hline $2_{\text {endo }}$ & 215.7 & 134.3 & 25.9 & 157.9 & 100.7 \\
\hline $2_{e p i}$ & 197.3 & 149.7 & 129.3 & 149.3 & 120.0 \\
\hline $3_{\text {endo }}$ & 144.5 & 115.8 & 9.1 & 111.5 & 87.2 \\
\hline $3_{\text {epi }}$ & 281.7 & 281.7 & 25.0 & 215.3 & 77.2 \\
\hline$M E A N_{\text {endo }}$ & 179.1 & 104.0 & 63.5 & 128.9 & 114.3 \\
\hline$M E A N_{e p i}$ & 265.5 & 226.2 & 78.7 & 215.7 & 148.6 \\
\hline$M E A N_{C A R T O}$ & 222.3 & 165.1 & 71.1 & 172.3 & 131.5 \\
\hline
\end{tabular}

Table 1. Spatial dispersion of ARI across the endoand epicardium walls in segments defined by the AHA 17-segment left ventricule model for each patient in the CARTO dataset, and the mean for each region.

\begin{tabular}{|c|c|c|c|c|c|}
\hline 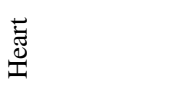 & $\frac{\frac{0}{0}}{\frac{2}{3}}$ & 1 & 6 & 7 & 12 \\
\hline $1_{\text {endo }}$ & 93.2 & 88.9 & 44.9 & 57.0 & 65.4 \\
\hline $1_{e p i}$ & 164.3 & 57.4 & 48.3 & 137.2 & 98.1 \\
\hline $2_{\text {endo }}$ & 114.3 & 84.5 & 40.1 & 45.5 & 111.1 \\
\hline $2_{e p i}$ & 74.0 & 37.8 & 5.0 & 57.3 & 20.9 \\
\hline $3_{\text {endo }}$ & 148.3 & 97.8 & 34.3 & 103.5 & 88.8 \\
\hline $3_{\text {epi }}$ & 91.3 & 29.0 & 28.7 & 34.0 & 86.5 \\
\hline $4_{\text {endo }}$ & 184.5 & 97.6 & 81.1 & 85.5 & 184.5 \\
\hline $4_{e p i}$ & 303.2 & 240.0 & 143.0 & 174.3 & 246.4 \\
\hline$M E A N_{\text {endo }}$ & 135.1 & 92.2 & 50.1 & 72.9 & 112.5 \\
\hline$M E A N_{e p i}$ & 158.2 & 91.1 & 56.2 & 100.7 & 113.0 \\
\hline$M E A N_{O P T}$ & 146.6 & 91.6 & 53.2 & 86.8 & 112.7 \\
\hline
\end{tabular}

Table 2. Spatial dispersion of APD across the endo- and epicardium walls in segments defined by the AHA 17segment left ventricule model for each heart in the optically recorded dataset, and the mean for each region.

\section{Discussion}

Clearly a major limitation of this study is the availability of datasets. With only three CARTO datasets and four optical datasets, and between them only six common regions, it is hard to make quantifiable results. In addition, the region of interest in this study is a very small region of the left ventricle, despite having ARI data for the entire ventricular mesh. Since so much data is left redundant, clearly a better method of validation is required that can encompass the entire mesh. However, the distance weighted interpolation and the use of UVCs has allowed for the CARTO and optical data to be described in a common frame that allows for their direct comparison.

We are able to show the validity of ARI as a surrogate for APD, but we are not able to directly compare the repolarization times from the two samples despite having the data. Since the optically recorded heart slices are ex- 


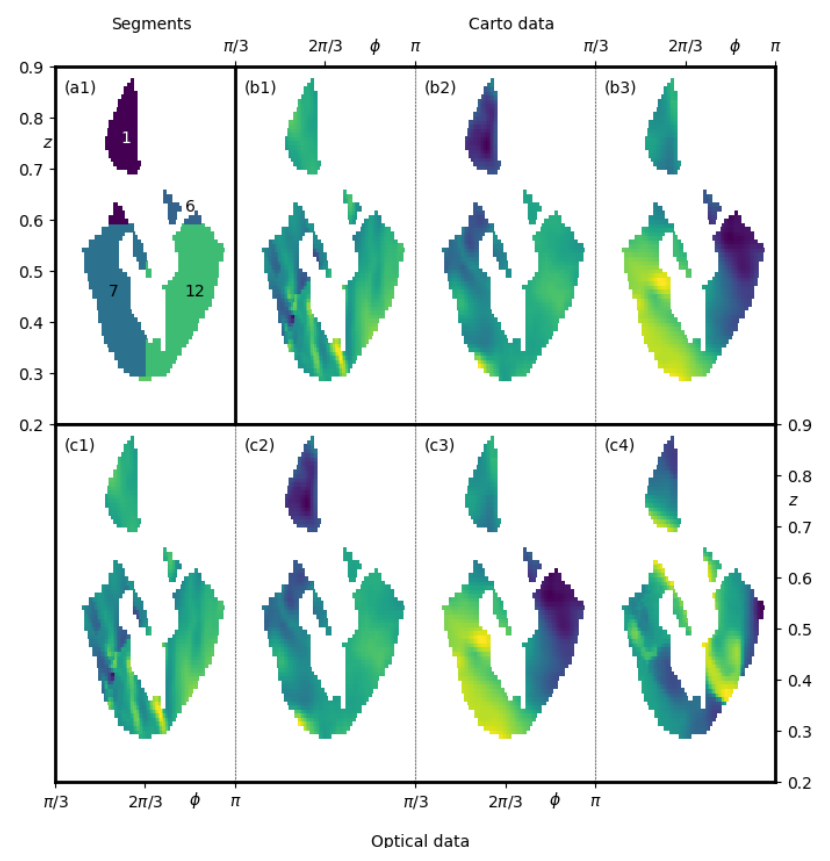

Figure 2. (a) Portion of left ventricle epicardium in UVC $(\phi, z)$-coordinates, with AHA 17-segment left ventricle plan segments labeled. APD (b) and ARI (c) data from optical and CARTO datasets, respectively, mapped to the portion of LV shown in (a).

planted slices, the activity in each of slices was manually triggered by a point stimulus in an attempt to recreate the natural conduction. This is unlikely to be representative of reality, and so activation and repolarization times associated with these slices could not be used reliably. However, the APD calculations are local calculations and are independent of factors such as the order in which regions activate. Furthermore, the ongoing debate over how to extract ARI from unipolar signals tends to focus more on what feature of the signal corresponds to the moment of repolarization of the underlying action potential, and that the moment of activation seems to be generally agreed to be the point of maximal negative slope of the depolarization phase. Hence, showing that ARI is a good surrogate for APD, implies that the estimations of repolarization time are reliable.

\section{Conclusions}

This study has demonstrated that spatial dispersion of ARI extracted from unipolar recordings is consistent with that of optically recorded APD in regions of left ventricle. In addition, the spatial dispersion was compared between sub-regions of the ventricle as described by the AHA. This shows the local agreement of ARI and APD between the two sets of data. Finally, it provides an example of how
UVCs can be used to allow direct comparison of ventricular data from two different sources.

These results support the notion that ARI is a good surrogate for APD, which in turn strengthens the validity of the calculation of repolarization time from unipolar recordings. This works towards more accurate investigation into spatial arrangement of repolarization maps and assessment of repolarization abnormalities. This is a step towards building spatially and temporally accurate patient-specific electrophysiological models, that give a detailed overview of patient's ventricular activity, without the need for invasive surgery.

\section{Acknowledgments}

This project has received funding from the Fondation Leducq (Research Grant number 16 CVD 02).

The research leading to these results has received funding from the People Programme (Marie Curie Actions) of the European Unions Seventh Framework Programme (FP7/2007-2013) under REA grant agreement n. PCOFUND-GA-2013-609102, through the PRESTIGE programme coordinated by Campus France.

\section{References}

[1] Han J, Moe GK. Nonuniform recovery of excitability in ventricular muscle. Circulation Research 1964;14(1):44-60.

[2] Yue AM, Paisey JR, Robinson S, Betts TR, Roberts PR, Morgan JM. Determination of human ventricular repolarization by noncontact mapping: Validation with monophasic action potential recordings. Circulation 2004;110(11):1343-1350.

[3] Wyatt R, Burgess M, Evans A, Lux R, Abildskov J, Tsutsumi T. Estimation of ventricular transmembrane action potential durations and repolarization times from unipolar electrograms. American Journal of Cardiology 1981;47:488.

[4] Bayer J, Prassl AJ, Pashaei A, Gomez JF, Frontera A, Neic A, Plank G, Vigmond EJ. Universal ventricular coordinates: A generic framework for describing position within the heart and transferring data. Medical image analysis 2018;45:8393.

[5] on Myocardial Segmentation AHAWG, for Cardiac Imaging: R, Cerqueira MD, Weissman NJ, Dilsizian V, Jacobs AK, Kaul S, Laskey WK, Pennell DJ, Rumberger JA, Ryan $\mathrm{T}$, et al. Standardized myocardial segmentation and nomenclature for tomographic imaging of the heart: a statement for healthcare professionals from the cardiac imaging committee of the council on clinical cardiology of the american heart association. Circulation 2002;105(4):539-542.

Address for correspondence:

Peter Langfield

IHU Liryc - Hôpital Xavier Arnozan, Avenue du Haut Lévêque, 33604 Pessac cedex, France.

Peter.Langfield@IHU-Liryc.fr 